Neurosurg Focus 27 (2):E4, 2009

\title{
Tumor-associated epilepsy
}

\author{
Kiran F. Rajneesh, M.D., M.S., ${ }^{1}$ And Devin K. Binder, M.D., Ph.D. ${ }^{1,2}$ \\ Departments of ${ }^{1}$ Neurological Surgery and ${ }^{2}$ Anatomy and Neurobiology, University of California, Irvine, \\ California
}

\begin{abstract}
Tumor-associated epilepsy is an important contributor to morbidity in patients with brain tumors. Proposed pathophysiological mechanisms to explain these effects range from neuronal and glial dysfunction to deranged vascular homeostasis, to ionic and $\mathrm{pH}$ changes. Perilesional tissue alterations play a vital role in the generation of tumor-associated seizures. Clinical studies have determined that tumor-associated seizures are usually focal with secondary generalization and often resistant to antiepileptic drugs. Tumor histopathological characteristics and location are independent factors that impact seizure burden. Further understanding of the mechanisms of tumor-associated epilepsy may lead to new types of treatments targeted at perilesional tissue alterations. (DOI: 10.3171/2009.5.FOCUS09101)
\end{abstract}

KEY WORDS $\bullet \quad$ brain tumor $\underset{\text { epilepsy }}{\bullet}$ • seizure
tumor-associated seizure $\bullet$ pathophysiology

$\mathrm{E}$ PILEPSY associated with PBTs causes seizures in $20-45 \%$ of patients, and tumor-associated epilepsy significantly decreases quality of life. ${ }^{20}$ These seizures typically manifest as focal seizures with secondary generalization and are commonly refractory to antiepileptic drug treatment. The underlying pathophysiological characteristics of seizures secondary to brain tumors are poorly understood. However, a variety of hypotheses have been proposed, including altered neuronal regulation and connections, deranged vascular permeability, abnormal BBB, and impaired glial cell function. The tumor itself may be the seizure focus, or the tumor may cause secondary perilesional tissue alterations such as growth, inflammation, edema, or necrosis, thereby triggering seizure activity. In this review, we will examine some of the clinical and basic science evidence regarding tumor-induced epilepsy.

\section{Tumor Histological Characteristics and Location}

The histological characteristics of tumors influence their propensity to generate seizures. Low-grade PBTs grow slowly, invade normal surrounding tissues, and have a high frequency of epilepsy. For example, DNETs and Grade II astrocytomas exhibit this kind of activity. ${ }^{10,14}$ Glioneuronal malformations such as gangliogliomas and focal cortical dysplasias lack normal neuronal organization and connections, and can form epileptic foci. ${ }^{31}$ High-grade gliomas like glioblastoma multiforme less frequently cause seizures..$^{10,14}$ The seizures generated by

Abbreviations used in this paper: $\mathrm{BBB}=$ blood-brain barrier; DNET $=$ dysembryoplastic neuroepithelial tumor; FCD $=$ focal cortical dysplasia; $\mathrm{GABA}=\gamma$-aminobutyric acid; LEAT $=$ long-term epilepsy-associated tumor; $\mathrm{PBT}=$ primary brain tumor. these lesions may originate in areas of necrosis and hemosiderin deposition..$^{29}$

Tumor location also plays a critical role. Both intraand extraaxial primary brain tumors may be associated with seizures. A variety of mechanisms may contribute to seizure generation: extraaxial tumors compress normal brain tissue, whereas intraaxial tumors infiltrate normal brain tissue. Intraaxial location may also influence epileptogenicity, with temporal or frontal lobe location being associated more with seizures than lesions in other lobes. ${ }^{20,23,38,39}$ Infratentorial and sellar tumors seldom cause seizures. ${ }^{20}$

\section{Peritumoral Homeostasis Imbalance}

Alterations in the peritumoral brain microenvironment may predispose to seizure generation. Some of the common changes seen in patients with PBTs are altered BBB function, enzymatic changes, and impaired intercellular connections. Alteration or disruption of the BBB may lead to abnormal leakiness and extravasation of plasma proteins or other substances, which may lead to increased excitability and seizures. For example, it is known that focal disruption of the BBB can lead to the development of a seizure focus. ${ }^{16}$ The peritumoral microenvironment is also associated with altered levels of enzymes such as lactate dehydrogenase, adenosine 3',5'-cyclic monophosphate (cAMP) phosphodiesterase, enolase, and thymidine kinase, which may cause metabolic imbalance. ${ }^{17}$ Intercellular connections between adjacent glial cells occur via connexin transmembrane gap junction proteins. ${ }^{1}$ Altered expression of connexins has been found in epilepsy-associated brain tumors, ${ }^{1}$ and may predispose the perilesional epileptic cortex to hyperexcitability.

Other alterations in the peritumoral microenviron- 
ment may contribute to seizure generation. Primary brain tumors have a relatively higher metabolic rate than normal brain tissue. This creates a relative hypoxia and interstitial acidosis. Marked vasogenic brain edema is commonly seen around brain tumors, and increased tissue water content may also modulate neural excitability. ${ }^{33}$ In addition to increased water content, an increase in sodium, calcium, and serum proteins has been seen in quantitative studies of peritumoral tissue. ${ }^{15}$

\section{Neurotransmitters, Receptors, and Amino Acids}

Brain tumors have been found to have altered expression of neurotransmitter receptors. Of particular importance to seizure generation are the ionotropic and metabotropic glutamate receptors. Gliomas exhibit a high concentration of ionotropic glutamate receptors $(\alpha$-amino3-hydroxyl-5-methyl-4-isoxazole-propionate [AMPA], $\mathrm{N}$ methyl-D-aspartic acid [NMDA], and kainate receptors). In one study, the authors demonstrated altered editing of glutamate receptor subunit B in malignant gliomas, potentially contributing to hyperexcitability. ${ }^{25}$ Alternatively, sustained NMDA receptor activation may represent a pathological mechanism for seizure generation. ${ }^{26}$ Astrocytes in perilesional areas demonstrate increased expression of kainate receptors. ${ }^{3}$ Activation of kainate receptors has been demonstrated to downregulate inhibitory stimuli, which may predispose to formation of an epileptic focus in the perilesional area. ${ }^{30}$ Metabotropic glutamate receptors lead to intracellular signaling through guanosine triphosphate-related proteins and protein kinase cascades, resulting in long-term neuromodulation. ${ }^{3}$ Multiple subtypes of metabotropic glutamate receptors have been found to be overexpressed in reactive astrocytes in the perilesional zone around tumors compared with normal cortex. ${ }^{3}$

Glutamate, the major excitatory amino acid neurotransmitter in the brain, may contribute to perilesional seizure generation. One recent hypothesis of tumor invasion proposes that glioma cells use the release of glutamate to induce excitotoxic destruction of neurons to create new micropassages for migration of tumor cells..$^{34,35}$ Neoplastic cells have inherently higher glutamate concentrations that serve as an autocrine trigger to increase the rhythmic oscillations of calcium usually necessary for cellular migration. ${ }^{24}$ These intrinsically higher glutamate concentrations may also lead to local hyperexcitability along the paths of glioma cell migration.

Alterations in GABA, the main inhibitory neurotransmitter in the brain, may also contribute to tumor-associated seizures. It would be simplistic to assume that increased GABA activity would always suppress epileptic activity; GABA does not always hyperpolarize neurons. Based on the GABA reversal potential (itself determined by expression of chloride transporters), GABA can depolarize neurons. ${ }^{18,19,27}$ Studies of PBTs demonstrate increased GABA immunoreactivity, ${ }^{4,13}$ but further studies will be necessary to determine the exact role of the GABA system in contributing to the hyperexcitability of perilesional areas.

\section{Glioneuronal Tumors and Seizures}

Glioneuronal tumors such as gangliogliomas are strongly associated with seizures..$^{5,8,22,28}$ These lesions contain both a highly differentiated glial component and also a dysplastic neuronal component. Gangliogliomas are the most frequent tumor in patients with focal epilepsy. ${ }^{8}$ Another subtype within glioneuronal tumors are DNETs composed of floating neurons and oligodendroglia-like elements. ${ }^{12,41}$ These lesions are on a continuum with types of FCDs associated with epilepsy, ${ }^{36}$ the main distinction being that FCDs have no proliferative component. ${ }^{28}$ The exact cellular and molecular reasons why glioneuronal tumors are associated with epilepsy are not completely clear. Dysplasia-like neuronal/glial disorganization is one factor. Biochemical alterations in phosphatidylinositol 3-kinase pathway components in epilepsy-associated glioneuronal lesions have also been found. ${ }^{31}$ In addition, glioneuronal tumors overexpress neurotransmitter producing enzymes, neurotransmitter receptors, and neuropeptides, all of which may contribute to the formation of a hyperexcitable focus ${ }^{40,42}$ An extensive perilesional inflammatory reaction with accumulation of activated microglial cells may also contribute to focal seizures arising from these lesions. ${ }^{2}$

\section{Selected Clinical Studies}

One of the largest series of tumor-associated epilepsy cases is the Bonn series. Between 1988 and 1999, 229 patients were treated for PBT associated with epilepsy. ${ }^{21}$ These patients had both pharmacoresistent epilepsy and a PBT. Inclusion criteria included intractable epilepsy for longer than 2 years, a supratentorial and hemispheric lesion location, presurgical evaluation, resection of $>$ $50 \%$ of the tumor, and the availability of postoperative MR imaging. Out of 229 patients, 207 were available for follow-up. The median duration of follow-up was 8 years (range 2-14 years). Histopathological analysis revealed $144(70 \%)$ WHO Grade I tumors (82 gangliogliomas, 33 pilocytic astrocytomas, and 29 DNETs), 59 (29\%) WHO Grade II lesions (35 astrocytomas, 15 oligodendrogliomas, 5 pleomorphic xanthoastrocytomas, and 4 gangliogliomas), and 4 (1\%) WHO Grade III tumors (3 astrocytomas and 1 ganglioglioma). Most tumors were in the temporal lobe $(83 \%)$.

Patients in this study had pharmacoresistent seizures for longer than 2 years, with median seizure onset at the age of 13 years, and underwent surgery at the median age of 28 years. Preoperative electroencephalography revealed an additional seizure focus other than the tumor in $13 \%$ of the patients. Two hundred and three patients $(97.5 \%)$ had complex partial seizures, $62 \%$ of which progressed to secondary generalization. One year after surgery, 169 (82\%) were seizure free (Engel Class I), 7 (3\%) had rare seizures (Engel Class II), 20 (10\%) had worthwhile improvement (Engel Class III), and 11 (5\%) had no benefit (Engel Class IV). In 67 (40\%) of the seizure-free patients, antiepileptic drugs could be discontinued. Epilepsy recurred in only $18(11 \%)$ of the 169 seizure-free patients. Eleven (29\%) of the 38 patients originally classified into Engel Classes II-IV eventually improved to Engel Class I at a median follow-up of 3 years.

Overall, the results of this large study demonstrate that most tumor-associated pharmacoresistent epilepsy 
occurs with lower-grade tumors, and that there is a temporal lobe predominance. These authors also found that improvement of seizure outcome after tumor resection was dependent on histological characteristics, complete resection of the tumor, and early tumor removal. Patient age older than 40 years, frontal tumors, gemistocytic differentiation, and incomplete resection were associated with poorer seizure outcome..$^{21}$

In a related study, the Bonn group presented evidence for a subtype of Grade II astrocytomas in long-term epilepsy with a different prognosis and unique histological characteristics. ${ }^{32}$ Two groups were compared: a group of patients with LEAT astrocytomas (19 patients) with a mean seizure duration of 12.5 years, and a second group of patients with ordinary astrocytomas (87 patients) with a mean seizure duration of 1.5 years. All patients underwent surgery between 1988 and 1999, and were followed up for 2-13 years (median 7 years). All tumor cases were reviewed and partly reclassified as a result of the use of modern immunohistochemical techniques. Histological subtyping revealed a possible new isomorphic astrocytoma in 7 patients. Cytological hallmarks consisted of low cellularity, lack of mitotic activity, and highly differentiated astroglial elements infiltrating into adjacent brain parenchyma. ${ }^{9}$ Compared with the classical variant of diffuse astrocytoma, immunohistochemical reactions revealed a cellular proliferation $<1 \%$, absence of nuclear p53 accumulation, and a lack of glial MAP2 and CD34 expression. Patients with the isomorphic subtype had $50 \%$ fewer recurrences at 7.5 years and an estimated long-term survival of $80 \%$. Patients with LEAT tumor astrocytomas differed from those with ordinary non-LEAT astrocytomas in overall length of history, younger age at first seizure, and a higher percentage of 10-year survivors (80\%). The authors postulated a new subtype of epilepsyassociated astrocytoma, provisionally called isomorphic LEAT astrocytoma, with a significantly better survival and lower recurrence rate.

In another large study of patients undergoing temporal lobe epilepsy surgery, some of whom had tumors, prognostic factors and outcomes were evaluated. ${ }^{11} \mathrm{~A}$ series of 321 patients underwent surgery for temporal lobe epilepsy between 1989 and 1997. The mean follow-up duration was 38 months. Of the 321 patients, only 312 had a clear histopathological diagnosis: 116 patients $(36.1 \%)$ had a neoplastic pathological entity, among which there were 56 gangliogliomas, 16 DNETs, and 44 other gliomas. Seizure outcome was better in patients with gangliogliomas or DNETs (94.4\% good seizure control) than in patients with other gliomas (79.6\% good seizure control), and least favorable in those with cortical dysplasia $(68.3 \%$ good seizure control).

Another study of epileptogenic parietal and occipital lobe lesions correlated MR imaging and histopathological characteristics with seizure control. ${ }^{37}$ In this retrospective study, 42 patients who underwent resective epilepsy surgery for parietal and occipital lobe lesions between 1998 and 2003 were analyzed. Histopathological diagnoses included 5 gangliogliomas, 5 DNETs, 16 FCDs, 4 vascular malformations, 6 gyral scars, and 1 infection. Postoperative seizure-free outcome differed by the cause of seizures: $62 \%$ seizure free for glioneuronal tumors, $69 \%$ for FCDs, $71 \%$ for vascular malformations, and $40 \%$ for gyral scars. Overall, this and other studies of parietal and occipital epilepsy have indicated a seizure-free outcome $>60 \%$ after resection of epilepsy-associated glioneuronal tumors.,

\section{Future Directions}

Tumor-associated epilepsy is an important clinical problem. The authors of clinical studies have suggested that intractable seizures are generally associated with early-onset and lower-grade lesions that may have more benign tumor histological characteristics but nevertheless cause chronic seizures. This is true of low-grade tumors such as oligodendrogliomas and low-grade astrocytomas, as well as glioneuronal tumors such as gangliogliomas and DNETs. After tumor resection, seizure freedom is usually excellent $(\sim$ two-thirds of patients are seizure free). Across all studies, the most significant factors associated with seizure freedom are completeness of tumor resection and preoperative duration of tumor-associated epilepsy.

The pathogenesis of tumor-associated seizures remains unclear. Various studies have implicated changes in amino acid neurotransmitters, neurotransmitter receptors, and perilesional tissue changes such as reactive astrocytosis, inflammation, and microglial proliferation. Recent studies have focused on derangements in the BBB and changes in glial cells in peritumoral tissue. Tumor cells themselves may create intrinsic epileptogenicity by virtue of glutamate secretion, and inadequate homeostasis in the peritumoral tissue may then lead to alterations in the excitation-inhibition balance, leading to seizure susceptibility. Other local changes in metabolism, electrolytes, and enzymes may also contribute. Increased understanding of the dynamic processes at the tumor-brain interface may lead to novel concepts and treatment targets for the control of tumor-associated epilepsy in the future.

\section{Disclaimer}

The authors report no conflict of interest concerning the materials or methods used in this study or the findings specified in this paper.

\section{References}

1. Aronica E, Gorter JA, Jansen GH, Leenstra S, Yankaya B, Troost D: Expression of connexin 43 and connexin 32 gapjunction proteins in epilepsy-associated brain tumors and in the perilesional epileptic cortex. Acta Neuropathol 101:449459, 2001

2. Aronica E, Gorter JA, Redeker S, Ramkema M, Spliet WG, van Rijen PC, et al: Distribution, characterization and clinical significance of microglia in glioneuronal tumours from patients with chronic intractable epilepsy. Neuropathol Appl Neurobiol 31:280-291, 2005

3. Aronica E, Yankaya B, Jansen GH, Leenstra S, van Veelen $\mathrm{CW}$, Gorter JA, et al: Ionotropic and metabotropic glutamate receptor protein expression in glioneuronal tumours from patients with intractable epilepsy. Neuropathol Appl Neurobiol 27:223-237, 2001

4. Bateman DE, Hardy JA, McDermott JR, Parker DS, Edwardson JA: Amino acid neurotransmitter levels in gliomas and 
their relationship to the incidence of epilepsy. Neurol Res 10:112-114, 1988

5. Becker AJ, Lobach M, Klein H, Normann S, Nothen MM, von Deimling A, et al: Mutational analysis of TSC1 and TSC2 genes in gangliogliomas. Neuropathol Appl Neurobiol 27:105-114, 2001

6. Binder DK, Podlogar M, Clusmann H, Bien C, Urbadch H, Schramm J, et al: Surgical treatment of parietal lobe epilepsy. Clinical article. J Neurosurg [epub ahead of print, February 6, 2009. DOI: 10.3171/2008.2.17665]

7. Binder DK, Von Lehe M, Kral T, Bien CG, Urbach H, Schramm J, et al: Surgical treatment of occipital lobe epilepsy. J Neurosurg 109:57-69, 2008

8. Blümcke I, Giencke K, Wardelmann E, Beyenburg S, Kral T, Sarioglu N, et al: The CD34 epitope is expressed in neoplastic and malformative lesions associated with chronic, focal epilepsies. Acta Neuropathol 97:481-490, 1999

9. Blümcke I, Luyken C, Urbach H, Schramm J, Wiestler OD: An isomorphic subtype of long-term epilepsy-associated astrocytomas associated with benign prognosis. Acta Neuropathol 107:381-388, 2004

10. Cascino GD: Epilepsy and brain tumors: implications for treatment. Epilepsia 31 (3 Suppl):S37-S44, 1990

11. Clusmann H, Schramm J, Kral T, Helmstaedter C, Ostertun B, Fimmers R, et al: Prognostic factors and outcome after different types of resection for temporal lobe epilepsy. J Neurosurg 97:1131-1141, 2002

12. Daumas-Duport C, Scheithauer BW, Chodkiewicz JP, Laws ER Jr, Vedrenne C: Dysembryoplastic neuroepithelial tumor: a surgically curable tumor of young patients with intractable partial seizures. Report of thirty-nine cases. Neurosurgery 23:545-556, 1988

13. Haglund MM, Berger MS, Kunkel DD, Franck JE, Ghatan $\mathrm{S}$, Ojemann GA: Changes in gamma-aminobutyric acid and somatostatin in epileptic cortex associated with low-grade gliomas. J Neurosurg 77:209-216, 1992

14. Herman ST: Epilepsy after brain insult: targeting epileptogenesis. Neurology 59 (9 Suppl):S21-S26, 2002

15. Hossmann KA, Seo K, Szymas J, Wechsler W: Quantitative analysis of experimental peritumoral edema in cats. Adv Neurol 52:449-458, 1990

16. Ivens S, Kaufer D, Flores LP, Bechmann I, Zumsteg D, Tomkins $\mathrm{O}$, et al: TGF-beta receptor-mediated albumin uptake into astrocytes is involved in neocortical epileptogenesis. Brain 130:535-547, 2007

17. Kim E, Lowenson JD, MacLaren DC, Clarke S, Young SG: Deficiency of a protein-repair enzyme results in the accumulation of altered proteins, retardation of growth, and fatal seizures in mice. Proc Natl Acad Sci U S A 94:6132-6137, 1997

18. Kohling R: Neuroscience. GABA becomes exciting. Science 298:1350-1351, 2002

19. Kohling R, Vreugdenhil M, Bracci E, Jefferys JG: Ictal epileptiform activity is facilitated by hippocampal GABAA receptor-mediated oscillations. J Neurosci 20:6820-6829, 2000

20. Liigant A, Haldre S, Oun A, Linnamagi U, Saar A, Asser T, et al: Seizure disorders in patients with brain tumors. Eur Neurol 45:46-51, 2001

21. Luyken C, Blumcke I, Fimmers R, Urbach H, Elger CE, Wiestler OD, et al: The spectrum of long-term epilepsy-associated tumors: long-term seizure and tumor outcome and neurosurgical aspects. Epilepsia 44:822-830, 2003

22. Luyken C, Blumcke I, Fimmers R, Urbach H, Wiestler OD, Schramm J: Supratentorial gangliogliomas: histopathologic grading and tumor recurrence in 184 patients with a median follow-up of 8 years. Cancer 101:146-155, 2004

23. Lynam LM, Lyons MK, Drazkowski JF, Sirven JI, Noe KH, Zimmerman RS, et al: Frequency of seizures in patients with newly diagnosed brain tumors: a retrospective review. Clin Neurol Neurosurg 109:634-638, 2007
24. Lyons SA, Chung WJ, Weaver AK, Ogunrinu T, Sontheimer $\mathrm{H}$ : Autocrine glutamate signaling promotes glioma cell invasion. Cancer Res 67:9463-9471, 2007

25. Maas S, Patt S, Schrey M, Rich A: Underediting of glutamate receptor GluR-B mRNA in malignant gliomas. Proc Natl Acad Sci U S A 98:14687-14692, 2001

26. Mody I, Heinemann U: NMDA receptors of dentate gyrus granule cells participate in synaptic transmission following kindling. Nature 326: 701-704, 1987

27. Palma E, Amici M, Sobrero F, Spinelli G, Di Angelantonio $\mathrm{S}$, Ragozzino D, et al: Anomalous levels of $\mathrm{Cl}^{-}$transporters in the hippocampal subiculum from temporal lobe epilepsy patients make GABA excitatory. Proc Natl Acad Sci U S A 103:8465-8468, 2006

28. Palmini A, Najm I, Avanzini G, Babb T, Guerrini R, FoldvarySchaefer N, et al: Terminology and classification of the cortical dysplasias. Neurology 62 (6 Suppl):S2-S8, 2004

29. Riva M: Brain tumoral epilepsy: a review. Neurol Sci 26 (1 Suppl):S40-S42, 2005

30. Rodriguez-Moreno A, Herreras O, Lerma J: Kainate receptors presynaptically downregulate GABAergic inhibition in the rat hippocampus. Neuron 19:893-901, 1997

31. Schick V, Majores M, Koch A, Elger CE, Schramm J, Urbach $\mathrm{H}$, et al: Alterations of phosphatidylinositol 3-kinase pathway components in epilepsy-associated glioneuronal lesions. Epilepsia 48 (5 Suppl):65-73, 2007

32. Schramm J, Luyken C, Urbach H, Fimmers R, Blumcke I: Evidence for a clinically distinct new subtype of grade II astrocytomas in patients with long-term epilepsy. Neurosurgery 55:340-348, 2004

33. Schwartzkroin PA, Baraban SC, Hochman DW: Osmolarity, ionic flux, and changes in brain excitability. Epilepsy Res 32:275-285, 1998

34. Sontheimer H: A role for glutamate in growth and invasion of primary brain tumors. J Neurochem 105:287-295, 2008

35. Sontheimer H: An unexpected role for ion channels in brain tumor metastasis. Exp Biol Med (Maywood) 233:779-791, 2008

36. Taylor DC, Falconer MA, Bruton CJ, Corsellis JA: Focal dysplasia of the cerebral cortex in epilepsy. J Neurol Neurosurg Psychiatry 34:369-387, 1971

37. Urbach H, Binder D, von Lehe M, Podlogar M, Bien CG, Becker A, et al: Correlation of MRI and histopathology in epileptogenic parietal and occipital lobe lesions. Seizure 16:608-614, 2007

38. van Breemen MS, Vecht CJ: Optimal seizure management in brain tumor patients. Curr Neurol Neurosci Rep 5:207-213, 2005

39. van Breemen MS, Wilms EB, Vecht CJ: Epilepsy in patients with brain tumours: epidemiology, mechanisms, and management. Lancet Neurol 6:421-430, 2007

40. Wolf HK, Birkholz T, Wellmer J, Blumcke I, Pietsch T, Wiestler OD: Neurochemical profile of glioneuronal lesions from patients with pharmacoresistant focal epilepsies. J Neuropathol Exp Neurol 54:689-697, 1995

41. Wolf HK, Buslei R, Blumcke I, Wiestler OD, Pietsch T: Neural antigens in oligodendrogliomas and dysembryoplastic neuroepithelial tumors. Acta Neuropathol 94:436-443, 1997

42. Wolf HK, Roos D, Blumcke I, Pietsch T, Wiestler OD: Perilesional neurochemical changes in focal epilepsies. Acta Neuropathol 91:376-384, 1996

Manuscript received April 14, 2009.

Accepted May 19, 2009.

Address correspondence to: Devin K. Binder, M.D., Ph.D., Department of Neurological Surgery, University of California, Irvine, 101 The City Drive South, Building 56, Suite 400, Orange, California 92868-3298. email: dbinder@uci.edu. 